\title{
Flexible and Efficient DSP-assisted Subcarrier Multiplexing for an Analog Mobile Fronthaul
}

\author{
Philippos Assimakopoulos, Member, IEEE, Shabnam Noor, Member, IEEE, Minqi Wang, Hazim \\ Abdulsada, Luiz Anet Neto, Naveena Genay, Philippe Chanclou and Nathan J. Gomes, Senior \\ Member, IEEE
}

\begin{abstract}
The digital formation of an analog subcarrier multiplex employing in combination both a technique using preIFFT frequency-domain samples and one using post-IFFT timedomain samples is proposed and demonstrated. This combined technique enables a compromise for sampling rate requirements, while maintaining low complexity and good performance.
\end{abstract}

Index Terms-Optical fiber communications, Subcarrier multiplexing, OFDM, 5G mobile communications, mobile fronthaul.

\section{INTRODUCTION}

L EGACY fronthaul has used industry standards, such as the Common Public Radio Interface (CPRI), to transport timedomain samples of radio waveforms between central units and remote radio units [1]. However, this approach is not scalable to the requirements of 5 th generation mobile networks $(5 \mathrm{G})$ and beyond due to the high bit-rates needed for wideband signals and the need to transport many signals for multi-antenna radio technologies [2]. As a result, new split-point interfaces in the Radio Access Network (RAN) have been defined by a number of bodies, including the 3rd Generation Partnership Project (3GPP) [3]; these involve moving some Physical (PHY) layer RAN functions to the remote unit, enabling the transport of frequency domain samples or protocol data units from higher parts of the PHY layer, depending on the precise split-point chosen [4]. While the new split-point interfaces reduce bit-rate requirements, there are latency constraints and the loss of centralization can inhibit the ability to, for example, jointly process signals [5].

Due to such constraints with the new RAN functional splits, the use of an analog fronthaul has been proposed, e.g. [6]. An analog fronthaul transports the radio waveforms (translated to different intermediate frequencies in a subcarrier multiplex to enable concurrent transport) between the central and remote units, offering the greatest degree of centralization. This form of transport makes use of the spectral efficiency of the radio signals in reducing the bandwidth requirements of the fronthaul optical link. However, analog transport suffers from some

Manuscript received xxx, 2020. This work was partly supported by the European Union Horizon 2020 project 5G-DRIVE (grant no. 814956).

S. Noor, P. Assimakopoulos and N.J. Gomes are with the Communications Research Group, University of Kent, Canterbury, CT2 7NT, UK (email: \{S.Noor, P.Asimakopoulos, N.J.Gomes\}@kent.ac.uk.). disadvantages: noise and distortion effects are cumulative and the reliance on microwave/RF components in the operation of the subcarrier multiplexing (SCM) has traditionally led to a lack of flexibility. For $5 \mathrm{G}$ and beyond fronthaul applications, with larger and variable channel bandwidths, variable numerologies, different bands of operation (including $\mathrm{mmW}$ ), flexibility and scalability will be of prime importance. Furthermore, the use of network slicing and orchestration techniques will require a fronthaul that can dynamically adapt its operation. Digital Signal Processing (DSP) techniques can be used to assist in the multiplex formation and offer greater flexibility and adaptability. A number of DSP-assisted multiplexing techniques have been investigated recently [7-11], some incorporating low-cost techniques for $\mathrm{mmW}$ up-conversion [8], [9]. While latency is not the focus in this work, it is noted that mobile fronthaul has stringent one-way latency requirements (less than $100 \mu \mathrm{s}$ if coordinated multi-point is employed [5]), and these approaches have achieved low latencies [7], [10], [11]; further reductions in latency can be achieved with filtering/windowing optimizations [7].

In previous work [9], it was proposed that SCM formation could be undertaken by using the pre-Inverse Fast Fourier Transform (pre-IFFT) frequency domain "samples" of each radio signal/channel: these samples are arranged appropriately (with null samples between them) and a single-IFFT operation then creates the time-domain SCM composite signal. This is termed the frequency-domain samples technique and is depicted in Fig.1(a). The frequency domain samples are "mapped" into contiguous groups of samples (called channels) in the frequency domain, prior to the IFFT operation. Arbitrary numbers of null samples (corresponding to null subcarriers) are inserted between these channels to form frequency-domain guard bands of arbitrary size. The frequency-domain multiplex is then sent to a single IFFT block where it is converted into the time-domain. This "single-IFFT" leads to the possibility of very large IFFT sizes, however, and increased sampling rates [10].

Recently, the technique of using the frequency domain samples with a "single-IFFT" operation has been compared with the technique of using the post-IFFT time domain samples

H. A. Abdulsada was with the Communications Research Group, University of Kent, Canterbury CT2 7NT, U.K. He is now with the Basra Oil Company (BOC)- Basra-Iraq (e-mail:haz3em@gmail.com).

M. Wang, L. Anet Neto, N. Genay and P. Chanclou are with the Access Networks Group, Orange Labs, Lannion, France (email: \{minqi.wang, luiz.anetneto, naveena.genay, philippe.chanclou $\} @$ orange.com). 

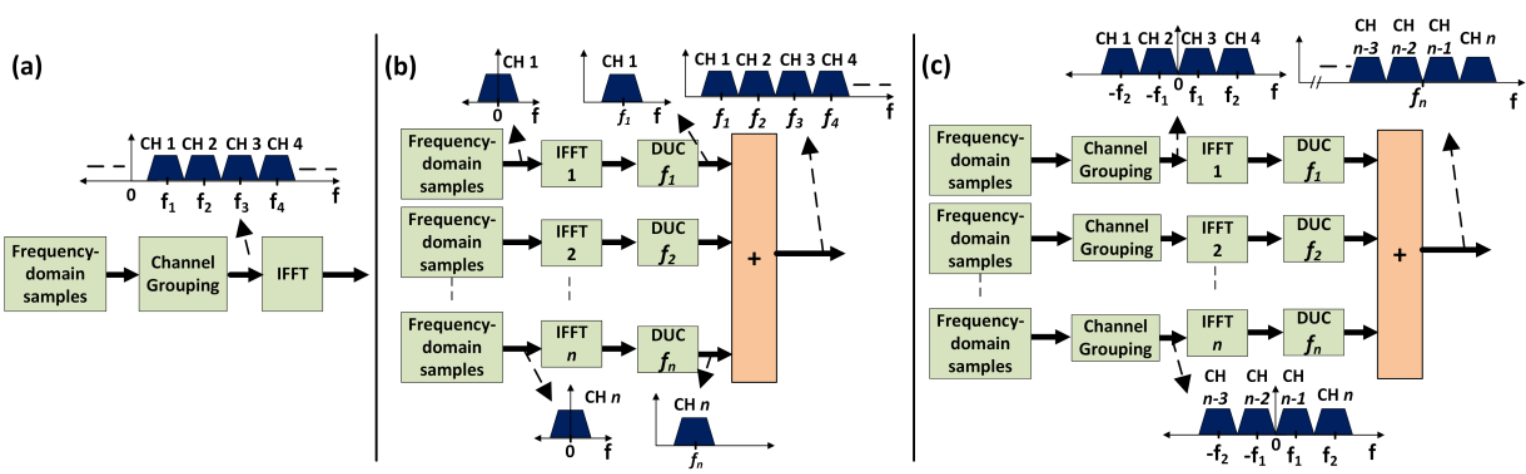

Fig. 1. Conceptual depiction of the different multiplexing techniques. (a) Frequency-Domain Samples technique. (b) Time-Domain Samples technique. (c) Combined technique. The $f_{1}, f_{2}, \ldots, f_{n}$, correspond to the DUC center frequencies. DUC, Digital UpConverter; IFFT, Inverse Fast-Fourier Transform; CH, Channel.

of each signal/channel to form the multiplex, again in the digital domain, through Digital Up-Converters (DUCs) [10]. This is termed the time-domain samples technique here and is depicted in Fig.1(b). Frequency-domain samples are again grouped into channels but each individual channel is sent to an IFFT block. The time-domain output from each IFFT block is then placed in its appropriate location within the multiplex by a DUC.

Despite the large IFFT size, it was shown that the technique using frequency domain samples possesses less computational complexity and generally leads to improved performance compared to that using the multiplexing of time domain samples. However, as higher sampling rates are often required with this technique [10], a combination of multiplexing techniques is a promising alternative. The investigation of such a combined technique is the focus of this paper. It should be noted that the processing functions used in these techniques ((I)FFTs, DUCs etc.) are common in the generation of both filtered (e.g. Filter-bank Multicarrier (FBMC)) [12], [13] and unfiltered OFDM variants [10]. A thorough complexity analysis for FBMC generation can be found in [12]. But, the use of the frequency-domain and time-domain samples techniques in combination, for fronthaul multiplexing, and informing their combination through a complexity analysis, is something that has not been reported in the literature.

In this paper, the use of this combined technique, as shown in Fig. 1(c), enabling realizable sampling rates and flexible multiplex creation and de-aggregation, is proposed and demonstrated. Here, whole groups of channels, grouped using the frequency-domain samples technique of Fig. 1a, are upconverted through a DUC to an appropriate frequency location within the multiplex. Note, that pre-IFFT, we do not employ conjugate symmetry and therefore the channels depicted in the positive side of the spectrum are independent from those in the negative side of the spectrum.

\section{COMPLEXITY AND SAMPLING RATE REQUIREMENTS}

Assuming SCM formation and disaggregation is carried out in the digital domain due to the desire for flexibility, the final (highest) sampling rate required will depend on the overall bandwidth of the multiplex. In [10], it was shown that if the multiplex formation can be carried out efficiently, then similar sampling rate requirements should result for both techniques. Divergence from this occurs due to the use of powers-of- 2 in the IFFT for the orthogonal frequency division multiplexing (OFDM) signals in 3GPP standards, including all new 5G standards up to now, and the desire to maintain powers-of- 2 for the IFFT for the composite multiplex. This is assumed to lead to the minimum computational complexity, highest speed, and optimum hardware implementation: the use of efficient, nonpowers-of-2 digital Fourier Transform processes are left for future investigation [14]. Under this assumption, the technique using frequency domain samples will be less efficient and require a higher sampling rate when the number of channels to be multiplexed is not a power-of- 2 or when the channel spacing between adjacent channels is large (necessitating the use of a higher power-of-2 IFFT).

Fig. 2 shows the sampling rate requirement for the three techniques, with annotations for the combined technique configurations. For example, if 12 channels are to be multiplexed, the time domain samples technique simply uses digital up-converters to up-sample from each channel, leading to a $24 \mathrm{x}$ (that of the individual channels) sampling rate. However, when frequency domain samples are used, the samples have to be arranged at the input of an IFFT with $32 x$ the number of samples of each channel, and a 32x sampling rate is required. This is where a combined approach can be helpful. If the 12 channels are arranged in three groups of 4 , each group can be multiplexed first using their frequency domain samples, and the time-domain samples from each of these IFFTs used to create the final multiplex. The resultant highest sampling rate remains at $24 \mathrm{x}$ that of the individual channels. Note that a number of combining options are possible, for example arranging 6 groups of 2 channels in the previous example. However more IFFTs require more DUCs, increasing the overall complexity. Therefore, the manner in which the techniques are combined is important. As a further example, a multiplex of 36 channels can be divided into 4 groups of 8 and 1 group of 4, with an overall sampling rate of $80 x$ that of each channel. Note that the individual groupings are chosen such that they can be multiplexed using efficient power-of-2 IFFTs. Using the frequency-domain samples technique in this case for the whole multiplex, would result in a sampling rate of $128 \mathrm{x}$ that of each channel, while a $72 x$ sampling rate would be needed for the time-domain samples technique.

While enabling sampling rate requirement reductions compared to the technique using only frequency domain 
samples, the combined technique also leads to some compromise in complexity. This complexity, measured as the number of multiplications per input sample (MPIS) is shown in Fig. 3, for all three techniques. The employed DUC interpolation factor is equal to twice the number of multiplexed channels (as indicated in the x-axis of the figure) or to the number of per-IFFT channel groupings for the combined technique. The channel parameters are based on 5G 3GPP specifications: Each channel has a bandwidth of approximately $100 \mathrm{MHz}$ and a subcarrier spacing of $120 \mathrm{kHz}$ while the individual channel sampling rate is $122.88 \mathrm{MHz}$. For the timedomain samples technique an IFFT length of 1024 is employed. For the time-domain samples and combined techniques, the filtering comprises of the interpolation section of the DUC consisting of a half-band filter, a Cascaded Integrator-Comb (CIC) compensator filter and a CIC interpolator. This design represents a typical filtering/interpolation section in a digital DUC (e.g., found in commercial SDRs) and all three filters are linear-phase Finite Impulse Response (FIR) implemented in a computationally efficient polyphase structure using the multirate algorithm available in MATLAB [10]. The complexity results shown in Fig. 3 for the combined technique correspond to the groupings shown as annotations in Fig. 2. For the combined technique, this corresponds to groupings of channels into as few IFFT processes as possible (thus having more

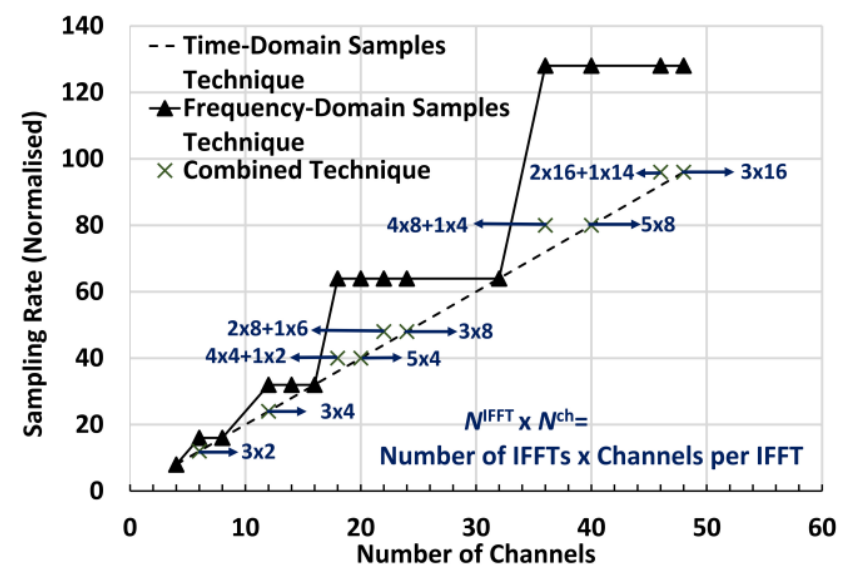

Fig. 2. Sampling rates (normalized to per channel sampling rate) versus number of channels in the final multiplex.

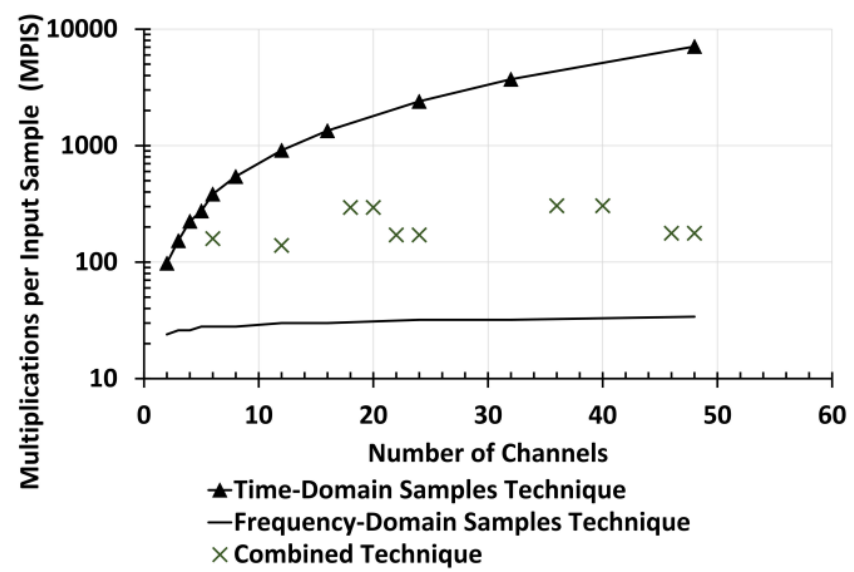

Fig. 3. Computational complexity given as number of Multiplications Per Input Sample (MPIS) versus number of channels in the final multiplex. channels per IFFT) and using the smallest number of DUCs. As the multiplex size increases, the complexity of the combined technique remains relatively low (compared to the time-domain technique) by progressively grouping more and more channels into each single-IFFT process.

\section{PERFORMANCE}

Fig. 4 shows the experimental set-up used to evaluate the proposed combined approach. Multiplex creation is performed in MATLAB through the generation of frequency-domain QAM samples, pilot insertion for tracking the channel frequency response, and multiplexing. The time-domain Inphase and Quadrature (I/Q) sampled signal is then downloaded into a Tektronix AWG7122C Arbitrary Waveform Generator (AWG), which performs digital-to-analog conversion. The AWG output is electrically amplified, applied to the MZM RF input and the modulated optical signal, after amplification through an Erbium Doped Fiber Amplifier (EDFA), is transmitted over a short-length fiber link (1 meter). The photodetected signal is amplified and captured with a Tektronix 72304DX DPO. The captured signal is processed offline in MATLAB with time-correction, de-multiplexing, per-channel FFT, frequency-domain equalization and demodulation, followed by EVM estimation. The de-multiplexing process for all three techniques is common and is carried out using direct Digital Down-Converters (DDCs).

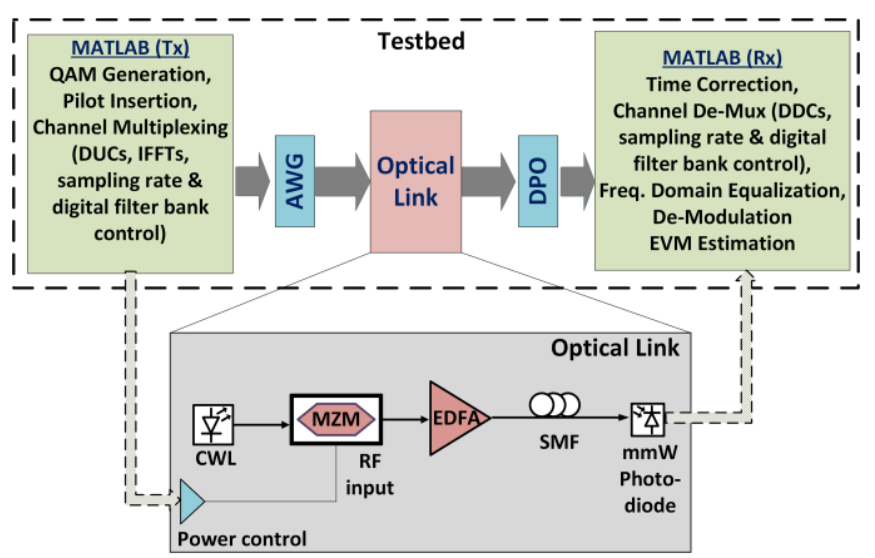

Fig. 4. Experimental setup for EVM measurements. AWG, Arbitrary Waveform Generator; DPO, Digital Phosphor Oscilloscope; CWL, Continuous Wave Laser; MZM, Mach-Zehnder Modulator; EDFA, Erbium Doped Fiber Amplifier; SMF, Single-Mode Fiber; mmW, millimeter wave.

Fig. 5 shows Error Vector Magnitude (EVM) results for the three multiplexing techniques, for narrow channel gaps of 0.5 $\mathrm{MHz}((\mathrm{a}),(\mathrm{b}))$ and wider channel gaps of $15 \mathrm{MHz}$ ((c), (d)). Fig. 5 (a) and (c) correspond to the baseline cases (i.e. back-to-back, no link) while (b) and (d) correspond to the cases with the optical link included. All channels have a subcarrier spacing of $120 \mathrm{kHz}$ while for the time-domain samples technique an IFFT length of 512 is employed. For the combined technique, a channel grouping of $3 \times 4$ is employed (that is, 3 IFFTs with 4 channels each). The EVM is given as the \% root-mean square (rms) value, averaged across all subcarriers and all channels within the multiplex, following the transmission of 10 frames 

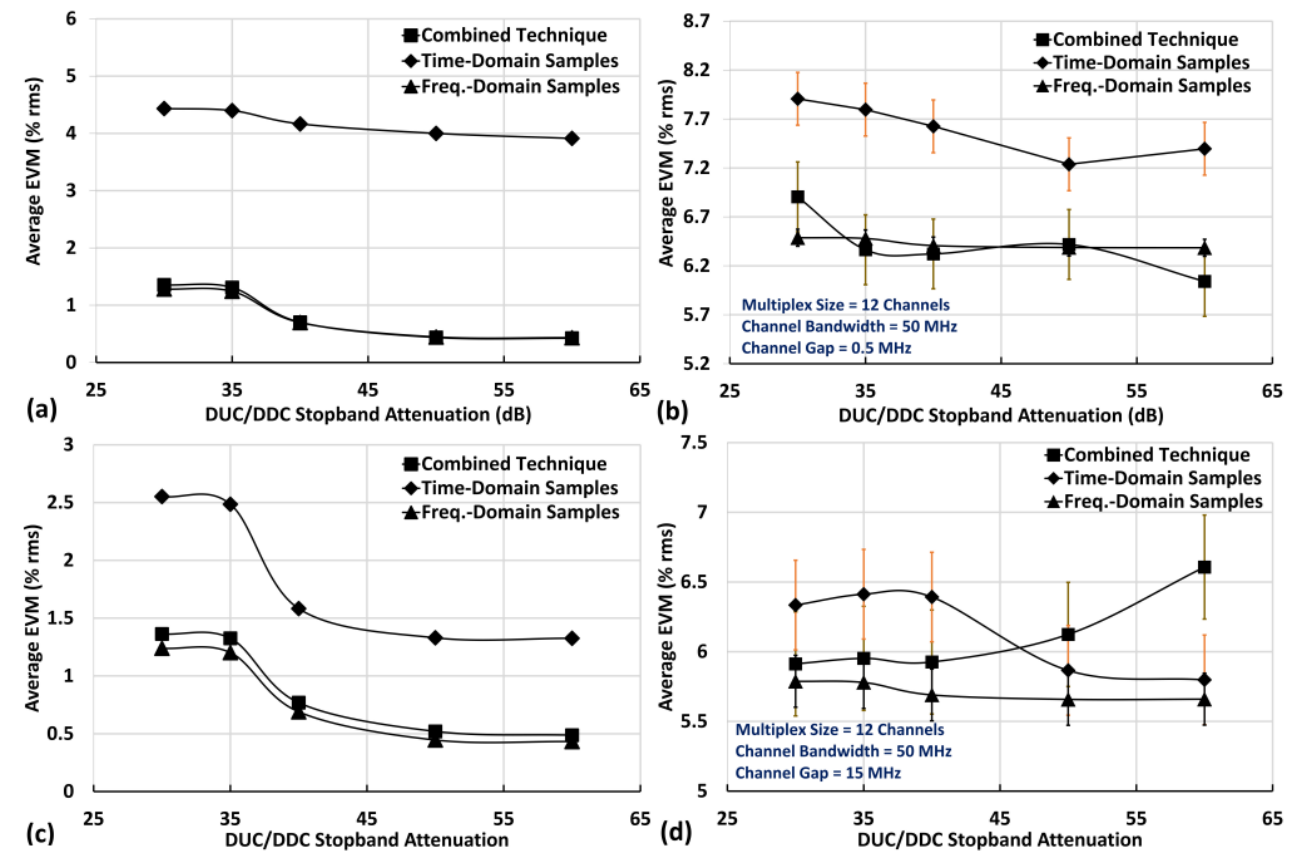

Fig. 5. EVM for different DUC/DDC stopband attenuations, for multiplex comprising twelve $50 \mathrm{MHz}$ channels. (a) Back-to-back case for channel gap of 0.5 MHz and (b) after transmission through optical link. (c) Back-to-back for channel gap of $15 \mathrm{MHz}$ and (d) after transmission through optical link. Note that for the frequency-domain samples technique there is no DUC employed at the transmitter (only a DDC at the receiver).

while in all cases 16-QAM modulation has been employed.

As reported in [10], in the baseline case, the time-domain technique exhibits worse EVM performance, as channels within the multiplex are not orthogonal (not formed in the same IFFT process). As the stopband attenuation increases, the EVM performance improves for both techniques, and both show improved performance with larger channel gaps (though more evident for the time-domain approach). The EVM performance of the combined technique is between that of the two extreme techniques but generally closer to that of the frequency-domain technique. This is expected due to the grouping of a number of channels into channel groups that are orthogonal. For the measured, experimental performance, the trends observed in the baseline case are not always clear due to the link-introduced noise floor. Still, the performance of the combined technique is comparable to that of the frequency-domain technique in all cases; there is negligible relative EVM degradation. While these results were obtained in a laboratory environment using high-cost equipment, implementation on hardware platforms such as field-programmable gate arrays will require further investigation on the effects of clock jitter, time-delay equalization (important also for addressing multiple RUs) and power imbalances between channels.

\section{CONCLUSION}

Subcarrier multiplex creation using a combination of techniques employing arrangement of frequency domain samples before an IFFT operation, and digital up-conversion of time domain samples after such operations, has been proposed and demonstrated. Appropriately combining the techniques, can balance sampling rate and complexity requirements, leading to hardware simplification while maintaining improved performance.

\section{REFERENCES}

[1] Common Public Radio Interface, Interface Specification 7.0., Oct. 2015 [Online]. Available at: www.cpri.info.html

[2] U. Dötsch et al., "Quantitative analysis of split base station processing and determination of advantageous architectures for LTE," Bell Labs Tech. J., vol. 18, no. 1, pp. 105-128, Jun. 2013

[3] 3GPP, "Study on new radio access technologies: Radio access architecture and interfaces,", 3rd Generation Partnership Project, Sophia Antipolis, France, Tech. Rep. TR 38.801, v14.0.0, 2017

[4] N.J. Gomes et al., "A flexible, Ethernet fronthaul for 5th generation mobile and beyond (Invited)," in Optical fiber Commun. Conf. (OFC), Anaheim, CA, 2016, paper W3C.1

[5] L. Larsen, A. Checko, and H.L. Christiansen, "A survey of the functional splits proposed for 5G mobile crosshaul networks," IEEE Comm. Surveys and Tutorials, vol.21, no.1, pp.146-172, 2019.

[6] N.J. Gomes et al., "The new flexible mobile fronthaul: Digital or analog, or both?" in Proc. Int. Conf. on Transparent Optical Networks (ICTON), Trento, Italy, July, 2016, pp. 1-4.

[7] X. Liu et al., "Efficient Mobile Fronthaul via DSP-Based Channel Aggregation," J. Lightw. Technol, Vol. 34, No. 6, pp. 1556-1564, Mar. 2016.

[8] N. Argyris, et al., "A 5G mmWave Fiber-Wireless IFoF Analog Mobile Fronthaul link with up to $24 \mathrm{~Gb} / \mathrm{s}$ Multi-band Wireless Capacity," $J$. Lightw. Technol., vol. 37, no. 12, pp. 2883-2891, Jun. 2019.

[9] S. Noor, P. Assimakopoulos, and N.J. Gomes, "Flexible Subcarrier Multiplexing System with Analog Transport and Digital Processing for 5G (and beyond) Fronthaul," J Lightw. Technol., vol. 37, no.14, pp.3689-3700, May. 2019.

[10] S. Noor et al., "Comparison of Digital Signal Processing Approaches for Subcarrier Multiplexed 5G and Beyond Analog Fronthaul," IEEE/OSA J. Optical Commun. Networking, vol. 12, no. 3, pp. 62-71, Mar. 2020.

[11] K. Tanaka et al., "First Experimental Demonstration of 5G Mobile Fronthaul Consisting of Cascaded IF-Over-Fiber Links, Frequency Converters and a Channel Selector," in Proc. European Conf. on Optical Commun. (ECOC), Rome, Italy, 2018, pp. 1-3.

[12] D. Demmer et al., "Study of OFDM Precoded Filter-Bank Waveforms," IEEE Trans. Wireless Commun., vol. 18, no. 6, pp. 2889-2902, Jun. 2019.

[13] Y. Liu et al., "Waveform Design for 5G Networks: Analysis and Comparison," IEEE Access, vol. 5, pp. 19282-19292, Feb. 2017.

[14] K. Li, W. Zheng, K. Li, "A Fast Algorithm With Less Operations for Length- $N=q \times 2^{m}$ DFTs," IEEE Trans. Signal Process., vol. 63, no. 3, pp. 673-683, Feb. 2015. 